\title{
Altered expression of the mRNA stability factor HuR promotes cyclooxygenase- 2 expression in colon cancer cells
}

\author{
Dan A. Dixon, ${ }^{1,2}$ Neal D. Tolley, ${ }^{2}$ Peter H. King, ${ }^{3}$ L. Burt Nabors, ${ }^{3}$ Thomas M. McIntyre, ${ }^{2}$ \\ Guy A. Zimmerman, ${ }^{2}$ and Stephen M. Prescott ${ }^{1,2}$
}

${ }^{1}$ Department of Oncological Sciences,
${ }^{2}$ Eccles Program in Human Molecular Biology and Genetics, and Huntsman Cancer Institute, University of Utah,
Salt Lake City, Utah, USA
${ }^{3}$ Department of Neurology, University of Alabama, and Birmingham Veterans Administration Medical Center,
Birmingham, Alabama, USA

Address correspondence to: Dan A. Dixon, Department of Surgery,

Vanderbilt University Medical Center, Nashville, Tennessee 37232 USA.

Phone: (615) 322-5244; Fax: (615) 322-6174; E-mail: dan.dixon@mcmail.vanderbilt.edu.

Received for publication April 9, 2001, and accepted in revised form October 17, 2001.

Cyclooxygenase-2 (COX-2) expression is normally tightly regulated. However, constitutive overexpression plays a key role in colon carcinogenesis. To understand the molecular nature of enhanced $\mathrm{COX}-2$ expression detected in colon cancer, we examined the ability of the AU-rich element-containing (ARE-containing) $3^{\prime}$ untranslated region ( $3^{\prime} \mathrm{UTR}$ ) of COX-2 mRNA to regulate rapid mRNA decay in human colon cancer cells. In tumor cells displaying enhanced growth and tumorigenicity that is correlated with elevated COX-2, vascular endothelial growth factor (VEGF), and IL-8 protein levels, the corresponding mRNAs were transcribed constitutively and turned over slowly. The observed mRNA stabilization is owing to defective recognition of class II-type AREs present within the COX-2, VEGF, and IL-8 3'UTRs; c-myc mRNA, containing a class I ARE decayed rapidly in the same cells. Correlating with cellular defects in mRNA stability, the RNA-binding of trans-acting cellular factors was altered. In particular, we found that the RNA-stability factor HuR binds to the COX-2 ARE, and overexpression of HuR, as detected in tumors, results in elevated expression of COX-2, VEGF, and IL-8. These findings demonstrate the functional significance rapid mRNA decay plays in controlling gene expression and show that dysregulation of these trans-acting factors can lead to overexpression of COX-2 and other angiogenic proteins, as detected in neoplasia.

J. Clin. Invest. 108:1657-1665 (2001). DOI:10.1172/JCI200112973.

\section{Introduction}

There is considerable evidence that arachidonic acid metabolites, particularly prostaglandins, participate in both normal growth responses and in aberrant growth, including carcinogenesis (1). Cyclooxygenases (COX) are key enzymes in the production of prostaglandins, and overexpression of the inducible isoform, COX-2, occurs at multiple stages of colon carcinogenesis $(2,3)$. The connection between COX-2 expression and colon cancer has been further established in genetic studies demonstrating a dramatic suppression of intestinal polyposis in mice deficient for COX-2 (4) along with demonstration that nonsteroidal, anti-inflammatory drugs (NSAIDs) specific for COX-2 have chemopreventive effects on the incidence of colon tumor formation and progression (5).

Molecular events leading to the overexpression of the COX-2 protein in colon cancer have not been definitively characterized. Previous studies have clearly demonstrated increased levels of COX-2 mRNA in colorectal adenomas, adenocarcinomas, and colon cancer cell lines (6-9). However, concomitant increases in COX-2 protein and prostaglandins have not been detected in all colon neoplasia or cell lines (10-14). These results, supported by the finding of constitutive activation of the COX-2 promoter in colon cancer cell lines (7), imply that transcriptional activation of COX-2 is an early event in the initiation of a colon tumor (15), but that enhanced expression of COX-2 protein, which has been shown to be a key factor for tumor growth (16-18), requires alteration of another facet of regulation occurring on the posttranscriptional level.

Recently, we identified an AU-rich element (ARE) within the $3^{\prime}$ untranslated region (3'UTR) of COX-2 mRNA that confers posttranscriptional regulation of expression by controlling both mRNA decay and protein translation $(19,20)$. In this study, we demonstrate that defects in rapid mRNA decay occur in colon cancer cells overexpressing COX-2 protein and angiogenic growth factors and thus can contribute to enhanced cell growth and tumor formation in vivo. This defect in posttranscriptional regulation is associated with altered recognition of the class II-type ARE by cellular trans-acting factors. We show here that the RNA stability factor HuR specifically interacts with the COX-2 ARE and, moreover, provides evidence that misregulated association of this RNA-binding protein with ARE-containing transcripts promotes expression of 
growth-related genes that can influence the neoplastic and angiogenic potential of cancer cells.

\section{Methods}

Cell culture and tumor growth. HT29 and LoVo cells were purchased from American Type Culture Collection (ATCC; Manassas, Virginia, USA) and maintained in DMEM containing $10 \%$ FBS. For cell growth studies, $10^{3}$ cells were plated in 96-well plates and allowed to adhere in normal growth media. After 16 hours, 100 $\mu$ M NS-398 (Cayman Chemical, Ann Arbor, Michigan, USA) or an equal volume of carrier ethanol was added, and the medium was replaced every 2 days. Cell growth was monitored using a colorimetric CellTiter 96-well proliferation assay (Promega Corp., Madison, Wisconsin, USA) following the vendor's instructions. Tumor growth in athymic nude mice was conducted as described (18) except $2 \times 10^{6}$ cells resuspended in PBS were injected into the dorsal subcutaneous tissue.

Immunoblot, ELISA, and prostaglandin analysis. Washed cells were lysed in RIPA buffer (1× PBS, $1 \%$ Nonidet P-40, $0.5 \%$ sodium deoxycholate, $0.1 \%$ SDS), then cleared lysates $(50 \mu \mathrm{g})$ were denatured and separated by $10 \%$ SDS-PAGE. Protein content was determined using a bicinchoninic acid (BCA) protein assay with BSA as standard (Pierce Chemical Co., Rockford, Illinois, USA). After electrophoresis, proteins were transferred to PVDF membranes and probed with a polyclonal $\mathrm{Ab}$ against COX-2 (Santa Cruz Biotechnology Inc., Santa Cruz, California, USA) or monoclonal anti-HuR Ab (3A2) (21). Detection of the phosphorylation status of ERK $1 / 2$ and Akt was accomplished using polyclonal Ab's against phospho-p44/42 MAP kinase (Thr202/Tyr204) and phospho-Akt (Ser473), along with the respective phosphorylation state-independent Ab's purchased from Cell Signaling Technology (Beverly, Massachusetts, USA). Where indicated, blots were stripped and then probed with anti-human $\beta$-actin Ab (ICN Radiochemicals Inc., Irvine, California, USA) or HRP-conjugated anti-FLAG M2 Ab (Sigma Chemical Co., St. Louis, Missouri, USA). Detection and quantitation of blots were performed as described (19).

The expression of angiogenic factors VEGF and IL-8 was assayed in media from cultures of HT29 and LoVo cells. The concentrations of VEGF and IL-8 in the culture medium were measured using anti-human VEGF or anti-human IL-8 Ab's by ELISA, according to the vendor's protocol (R\&D Systems Inc., Minneapolis, Minnesota, USA). PGE $_{2}$ levels in the media were determined by ELISA (Cayman Chemical) from cultures of HT29 and LoVo cells pretreated with $10 \mu \mathrm{M}$ NS-398 or carrier for 1 hour, after which the medium was removed and cells were incubated for 20 minutes with serum-free media containing $0.1 \%$ albumin and $20 \mu \mathrm{M}$ arachidonic acid (Cayman Chemical). Relative VEGF, IL-8, and $\mathrm{PGE}_{2}$ levels were normalized to total protein and are the average of triplicate experiments.

$m R N A$ analysis. RNase protection assays were used to detect COX-2, c-myc, and GAPDH mRNAs in $10 \mu \mathrm{g}$ of total RNA as described previously (19). COX-2 mRNA decay was assessed by Northern blot analysis using poly(A) RNA isolated with Oligotex beads (QIAGEN Inc., Valencia, California, USA) from $200 \mu \mathrm{g}$ of starting total RNA; similar decay results were seen using 20 $\mu \mathrm{g}$ of total RNA (data not shown). Samples were separated on formaldehyde-agarose gels and blotted onto nylon membranes that were probed with antisense riboprobes for COX-2, stripped, and then reprobed for GAPDH mRNA. VEGF and IL-8 mRNA decay was analyzed by RT-PCR using primers designed to amplify all human VEGF splice variants and a region of human IL-8 (221 bp) and control GAPDH (600 bp) from oligo(dT)-primed cDNA as described (19). In both HT29 and LoVo cells, VEGF 165 (444 bp) was the predominate transcript detected and was used to determine half-life. The expression of human HuR mRNA was detected similarly using primers designed to amplify the entire coding region (987 bp) along with a region of the control $\beta$-actin mRNA (514 bp).

ERK and Akt kinase assays. Determination of $\mathrm{p} 44 / 42$ MAP kinase and Akt kinase activities were accomplished using respective kits produced by Cell Signaling Technology. Actively growing HT29 and LoVo cells were lysed, and equal amounts of lysate were immunoprecipitated using monoclonal phospho-specific ERK1/2 or Akt Ab's; the immunoprecipitate was then incubated with ELK-1 or GSK-3 fusion proteins, respectively, in the presence of ATP. Phosphorylation of fusion proteins was detected by immunoblotting using $A b$ 's against phospho-ELK-1 (Ser383) or phospho-GSK-3 $\alpha / \beta$ (Ser21/9). Blots were quantitated digitally and presented as density units.

DNA transfections. Transient transfections of cells with lacZ+COX-2 3'UTR and Luc+COX-2 3'UTR reporter cDNA constructs (19) or a 1.8-kb human COX-2 promoter (7) cloned into pGL3-Basic (Promega Corp.) were conducted in six-well plates as described previously (19). Overexpression of HuR in LoVo cells was accomplished by transfecting pcDNA3-HuR-C-Flag (22) or empty pcDNA3 vector. Transfected cells were lysed in reporter lysis buffer (Promega Corp.) and assayed for $\beta$-galactosidase activity using Galacto-Light (Tropix, Bedford, Massachusetts, USA) or luciferase activity using the Luciferase Assay System (Promega Corp.). Reporter gene activities were normalized to cotransfected controls or total protein as indicated.

Immunohistochemistry. Tissue sections from paraffinembedded tumor samples (obtained from University of Alabama Tissue Procurement Office) were processed, and immunohistochemical staining of HuR was performed as described using a 1:120 dilution of purified rabbit anti-HuR Ab (23). Signal was developed using liquid diaminobenzidine tetrahydrochloride (DAB) and counterstained with hematoxylin I.

Analysis of protein-RNA interactions. Native gel mobility-shift assays and in vitro ultraviolet (UV) light crosslinking/label transfer experiments were conducted as described (19) to examine cytoplasmic protein binding 
to in vitro transcribed ${ }^{32} \mathrm{P}-$ labeled COX-2 ARE RNA. Immunoprecipitation of cross-linked HuR was accomplished as follows. After UV cross-linking and RNase digestion, $900 \mu \mathrm{l}$ of IP buffer (50 mM HEPES, pH 7.5, $150 \mathrm{mM} \mathrm{NaCl}, 5 \mathrm{mM} \mathrm{MgCl} 2,1 \% \mathrm{NP}-40,1 \mathrm{mM}$ DTT, $10 \%$ glycerol) was added, and reactions were incubated with equal amounts $(1 \mathrm{mg})$ of $\operatorname{IgG}$ obtained from of anti-Hu serum (24) or normal serum for 5 hours at $4{ }^{\circ} \mathrm{C}$. Protein A-agarose was added and immunoprecipitates were collected by brief centrifugation, washed four times with PBS, and electrophoresed in 10\% SDSPAGE. The ${ }^{32}$ P-labeled proteins were visualized by autoradiography and quantitated digitally.

Statistics. The data are expressed as the mean plus or minus standard deviation. Two-sample $t$ tests with equal variances were used to assess differences between means. $P$ values less than 0.05 were considered significant.

\section{Results}

Enhanced tumorigenicity and growth in cells with defective posttranscriptional control of COX-2 and angiogenic factor expression. A positive association of COX-2 expression with cell growth and tumorigenesis has been demonstrated in animal studies and cell culture systems $(4,13,25)$. To address the hypothesis that posttranscriptional mechanisms controlling rapid mRNA decay influence COX-2 expression and to identify potential cellular defects that allow for the unregulated COX-2 expression seen in neoplasia, human colon cancer cell lines (HT29, LoVo) were evaluated for differences in growth that correlate with COX-2 expression levels. To test this, equal numbers of HT29 and LoVo cells were implanted as xenografts into nude mice, and tumor growth was monitored. As shown in Figure 1a, after 30 days of tumor growth HT29 cells yielded highly vascular tumors (data not shown) six times larger than those derived from LoVo cell implants. The enhanced ability of HT29 cells to form tumors was also seen in the growth potential of HT29 cells in vitro. As with tumor growth, an increased rate of HT29 cell growth in culture was seen when compared with equal numbers of LoVo cells grown under identical conditions (Figure 1b). Marked growth inhibition was observed with both cells in the presence of the COX-2 selective inhibitor NS-398 suggesting growth to be dependent on the presence of COX-2, although under these experimental conditions COX2 -independent pathways can be influenced $(26,27)$. This inhibition of growth was not a result of the induction of apoptosis since under these cell culture conditions no cell death was seen at NS-398 concentrations ranging from 25 to $100 \mu \mathrm{M}$, whereas attenuation of cell growth was observed (data not shown).

To determine if the observed differences in cell growth characteristics correlated with the COX-2 protein levels, immunoblot and prostaglandin measurements in HT29 and LoVo cells were conducted. As anticipated, both cell lines constitutively express COX-2 protein, with HT29 cells expressing six- to sevenfold more protein than LoVo cells (Figure 1c). Respective COX activi- ty for each cell type reflected these findings (Figure 1d). Blockade of COX-2 activity with NS-398 left trace levels of $\mathrm{PGE}_{2}$ in both cell lines, indicating its synthesis is almost exclusively via COX-2 in these cells.

To determine if constitutive high levels of COX-2 protein in HT29 versus LoVo cells were reflected in a difference in their respective mRNA levels, steadystate mRNA levels were examined. As shown previously in other colon cancer cell lines (7), constitutive expression of COX-2 mRNA was observed in both cell types (Figure 2a). However, the level of COX-2 mRNA in HT29 cells was less than $20 \%$ of the mRNA found in LoVo cells. As a control, the level of proto-oncogene c-myc mRNA was examined and found to be essentially the same in the two cell types. A reporter construct containing a $1.8-\mathrm{kb}$ fragment of the human COX-2 promoter was used to show that the high level of COX-2 mRNA seen in LoVo cells stems from increased transcription (Figure $2 \mathrm{~b}$ ). No differences in expression of control reporter constructs lacking a promoter or containing an SV40 promoter were observed (data not shown). These findings suggest altered posttranscriptional regulation occurs in HT29
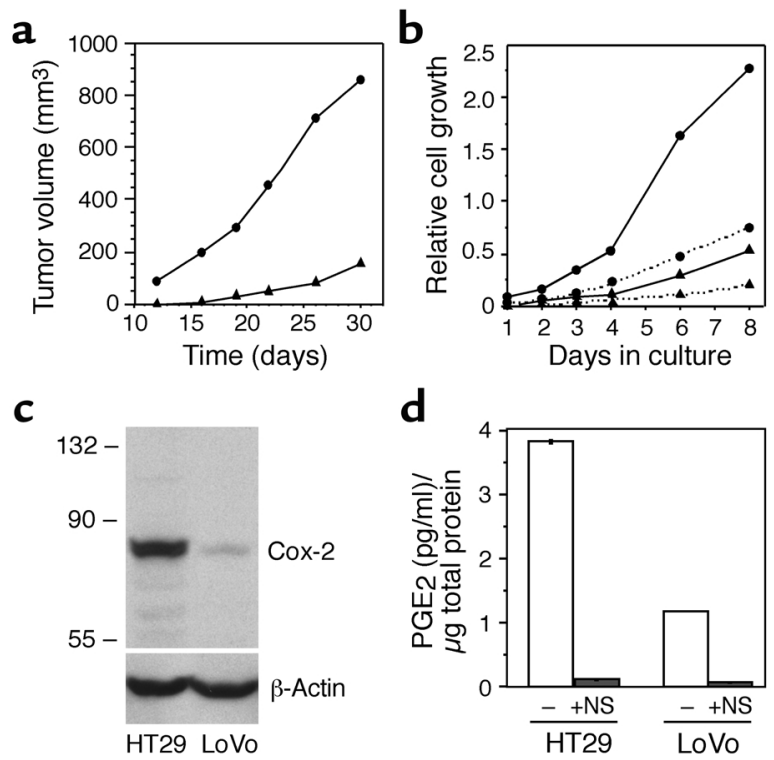

\section{Figure 1}

Enhanced growth and tumorigenicity correlates with COX-2 protein expression. (a) Tumor growth from HT29 and LoVo cell implants in nude mice was measured biweekly, and data shown are the mean tumor volume obtained from four xenografts each of HT29 cells (circles) and LoVo cells (triangles). (b) Growth curves of HT29 (circles) and LoVo (triangles) cells in culture under identical conditions. Growth in the presence of NS-398 is indicated with a dotted line. Each point represents the mean of four replicates. (c) Immunoblot of COX-2 protein in $50 \mu \mathrm{g}$ of HT29 and LoVo cell lysates. $\beta$-actin was detected on the same blot as a control for protein loading. Molecular-weight standards (kilodaltons) are listed on the left. The data shown represent three experiments. (d) COX activity in HT29 and LoVo cells treated with NS-398 (filled bars) or carrier (open bars). $\mathrm{PGE}_{2}$ levels were measured by ELISA in the media containing arachidonic acid and are the average of three experiments. 
a

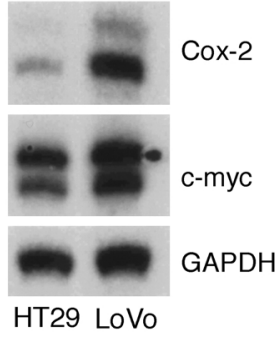

b

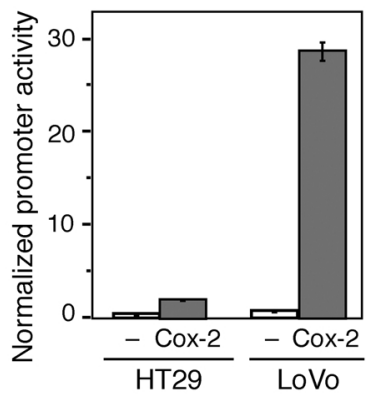

c

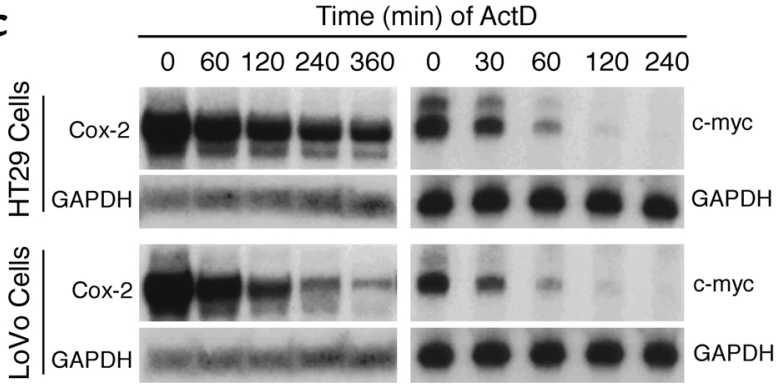

\section{Figure 2}

Posttranscriptional control of COX-2 mRNA. (a) Steady-state levels of COX-2 and c-myc mRNA in HT29 and LoVo cells were detected by RNase protection assays. The protected mRNA for c-myc was detected as a doublet. GAPDH mRNA is shown as a control for RNA loading. The data shown represent three experiments. (b) Levels of constitutive COX-2 transcription in HT29 and LoVo cells. A luciferase reporter construct containing the COX-2 promoter (filled bars) or the pGL3 vector (open bars) were cotransfected with the pSV- $\beta$ galactosidase control vector. Promoter activity was assessed as luciferase activity normalized to $\beta$-galactosidase activity and is the average of three experiments. (c) mRNA half-life experiments were initiated by adding $5 \mu \mathrm{g} / \mathrm{ml} \mathrm{ActD}$ to HT29 and LoVo cells for the indicated times and COX-2 mRNA decay was analyzed by Northern blot probing for COX-2 and the internal control GAPDH. Blot exposure times were adjusted to yield similar starting intensities of COX-2 mRNA. c-myc mRNA decay was analyzed similarly by RNase protection assay using riboprobes for c-myc and the internal control GAPDH. The data shown represent duplicate experiments.

cells, promoting enhanced synthesis of the COX-2 enzyme from low mRNA levels.

Normal cellular growth is associated with the rapid decay of COX-2 mRNA, whereas stabilization of COX-2 mRNA is seen in conditions that promote neoplastic growth $(19,20)$. To determine if HT29 cells are defective in this facet of posttranscriptional control, the decay of endogenous COX-2 mRNA was examined. The half-life of COX-2 mRNA was assessed by Northern blot after actinomycin $\mathrm{D}$ (ActD) was added to cells to halt transcription. The results shown in Figure $2 \mathrm{c}$ demonstrate that rapid COX-2 mRNA decay is seen in LoVo cells ( $t_{1 / 2}=44$ minutes), as previously observed with reporter genes containing the full-length COX-2 $3^{\prime}$ UTR or conserved AU-rich region (19). In contrast, COX-2 mRNA was stabilized approximately fivefold in HT29 cells $\left(t_{1 / 2}=210\right.$ minutes). As a comparison, the mRNA half-life of the constitutively expressed c-myc

was determined by RNase protection assay. Rapid turnover of c-myc mRNA was seen in both LoVo and HT29 cells ( $t_{1 / 2}=48$ and 42 minutes, respectively), suggesting that the defect in rapid mRNA decay seen in HT29 cells occurs for some, but not all mRNAs. To verify that the observed stabilization of COX-2 mRNA is not the result of gene mutations, we cloned and sequenced the exons containing the COX-2 coding region and $3^{\prime}$ UTR from both cells and found that there were no differences.

Based on the ability of COX-2 overexpression to stimulate the production of angiogenic growth factors (9, 18), we examined HT29 and LoVo cells for VEGF and IL-8 expression. As shown in Figure 3a, HT29 cells express approximately tenfold and twofold greater levels of VEGF $(P=0.0001)$ and IL-8 $(P=0.0143)$, respectively, than LoVo cells. To determine if alterations in rapid mRNA decay factored into the enhanced levels of VEGF and IL-8 seen with HT29 cells, the mRNA halflives of VEGF and IL-8 were examined by RT-PCR analysis (Figure $3 b$ ). Similar to the results shown in Figure $2 \mathrm{c}$ with COX-2, rapid VEGF and IL-8 mRNA decay was seen in LoVo cells $\left(t_{1 / 2}=51\right.$ and 70 minutes, respectively), whereas stabilization of both were detected in HT29 cells ( $t_{1 / 2}>360$ minutes).

Recent findings have demonstrated that activation of the mitogen-activated protein kinase (ERK) and
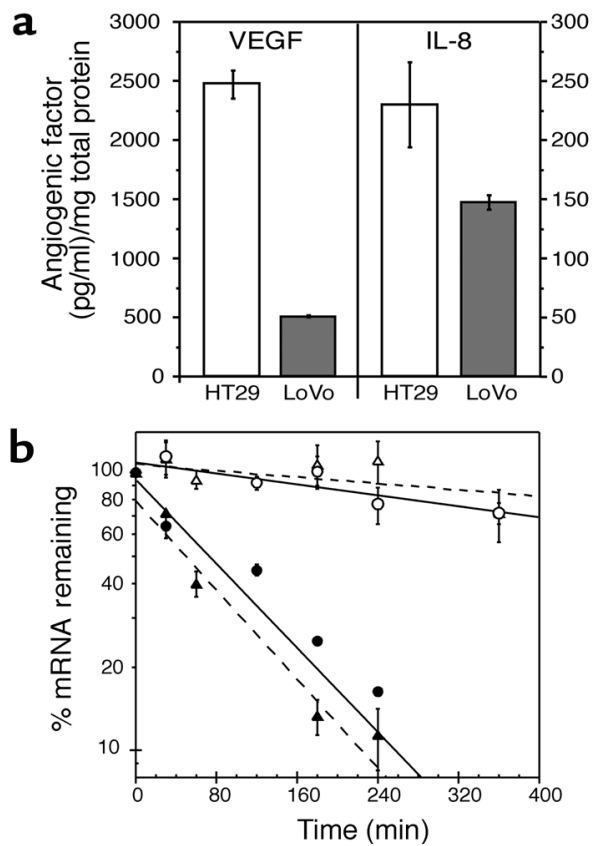

\section{Figure 3}

Posttranscriptional control of VEGF and IL-8 expression. (a) Expression of angiogenic factors VEGF (left panel) and IL-8 (right panel) in the culture media of HT29 (open bars) and LoVo cells (filled bars) were measured by ELISA and are the average of three experiments. (b) Decay curves of VEGF (triangles, hatched line) and IL-8 (circles, solid line) mRNAs from HT29 (open symbols) and LoVo cells (filled symbols) were obtained by RT-PCR analysis and normalized to the internal control GAPDH. The data shown is the average of three experiments. 

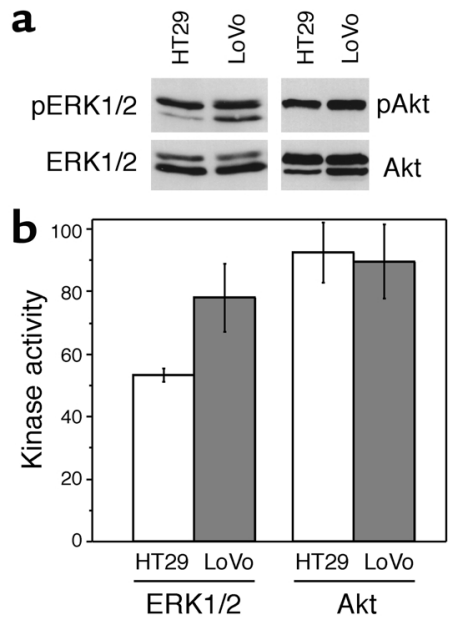

\section{Figure 4}

Constitutive activation of ERK and Akt/PKB pathways in HT29 and LoVo cells. (a) Equal amounts of HT29 and LoVo cell extracts (50 $\mu \mathrm{g})$ were examined for activation of ERKs and Akt/PKB through detection of phosphorylated ERK1/2 and Akt by immunoblot analysis. Blots were normalized to levels of total ERK1/2 and Akt and the data shown represent duplicate experiments. (b) ERK and Akt/PKB kinase assays. Equal amounts of HT29 and LoVo cell lysates $(200 \mu \mathrm{g})$ were immunoprecipitated using monospecific phospho-ERK $1 / 2$ or phospho-Akt Ab's. The immunoprecipitate was then incubated with ELK-1 or GSK-3 fusion proteins in the presence of ATP and kinase activity was detected by immunoblotting using Ab's against phospho-ELK-1 or phospho-GSK-3. Blots were quantitated digitally and kinase activity is presented as density units. The results shown are the average of duplicate experiments.

protein kinase B- signaling pathways (Akt/PKB-signaling pathways) are important in both the transcriptional and posttranscriptional regulation of COX-2 expression $(28,29)$. To determine if the observed mRNA stabilization detected in HT29 cells is a direct outcome of constitutive activation of either of these pathways, we examined the phosphorylation status of both ERK1/2 and Akt/PKB to assess activation of these pathways in HT29 and LoVo cells. Immunoblot analysis showed constitutive high levels of both activated ERK (phosphorylated ERK1/2) and Akt/PKB (phosphorylated at serine 473) in both cell lines (Figure 4a). In respective kinase activity assays (Figure 4b), LoVo cells had a slightly increased level of ERK1/2 kinase activity (1.5-fold) over HT29 cells; nearly identical levels of Akt/PKB kinase activity were detected in both cell lines (Figure 4b). Based on these results it appears that the observed stabilization of COX-2 detected in HT29 cells is not a direct result of constitutive activation of either of these pathways since LoVo cells, which display ERK and Akt/PKB kinase activation, show rapid mRNA decay. These findings, taken together, suggest that the mRNA stabilization observed in HT29 cells is a result of defective recognition of a cis-acting element that is common to COX-2, VEGF, and IL-8.

Altered recognition of the COX-2 AU-rich element. Posttranscriptional regulation of COX-2 expression has been shown to be mediated through the class II-type ARE-containing 3'UTR of COX-2 mRNA (19, 20, 28, 29). We postulated that defects in rapid mRNA decay in HT29 cells were a result of altered recognition of the COX-2 3'UTR by trans-acting regulatory factors. This hypothesis was tested by assaying the expression of transfected lac $Z$ reporter constructs containing the COX-2 3'UTR. As shown in Figure 5a, the COX-2 $3^{\prime}$ UTR inhibited $\beta$-galactosidase ( $\beta$-gal) expression in LoVo cells approximately twofold. In contrast, the COX-2 3'UTR did not inhibit expression in HT29 cells. Similar results were seen using constructs containing only the proximal 116-nucleotide ARE (data not shown), a region we had found previously to control COX-2 expression (19). These results imply that the ARE is essential for the regulation of mRNA stability. Since the reporter construct contained a wildtype COX-2 ARE, this result confirmed the earlier suggestion that a trans-acting factor is responsible for the altered regulation.

Several proteins with molecular weights ranging from 90 to $35 \mathrm{kDa}$ specifically bind to the COX-2 ARE (19). To determine if altered recognition of this element by cellular trans-acting regulatory proteins is responsible for the defective post-transcriptional control in HT29 cells, native gel mobility-shift and UV-cross-linking assays to examine factors binding the ARE were performed. In LoVo cells, stable protein-RNA complexes were seen (Figure 5b). HT29 cells, however, showed a very different pattern of ARE-binding, resulting in a heterogeneous smear. Defective ARE binding in HT29
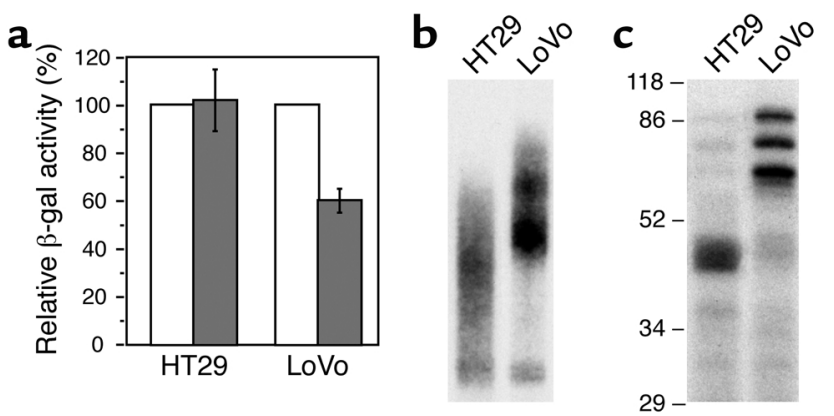

Figure 5

Altered recognition of the COX-2 3'UTR in HT29 cells.(a) LacZreporter gene expression constructs containing the full-length COX-2 3'UTR (filled bars) or no 3'UTR (open bars) were transfected in HT29 and LoVo cells. Transfection efficiency was controlled by cotransfection of the $\mathrm{pGL2-control} \mathrm{vector} \mathrm{expressing} \mathrm{luciferase}$ enzyme. Relative activity was assessed as $\beta$-galactosidase activity normalized to luciferase activity. All percentages listed are based on expression of $\beta$-galactosidase from the construct containing no $3^{\prime} \mathrm{UTR}$ and is the average of three experiments done in triplicate. (b and c) Cytoplasmic protein complexes from HT29 and LoVo cells bound to the radiolabeled COX-2 ARE were analyzed by native gel mobility-shift assay (b) or were cross-linked by UV-light irradiation, treated with RNase, and separated by $10 \%$ SDS-PAGE (c). Molecular-weight standards (kilodaltons) are listed on the left. The data shown are representative of three experiments. 


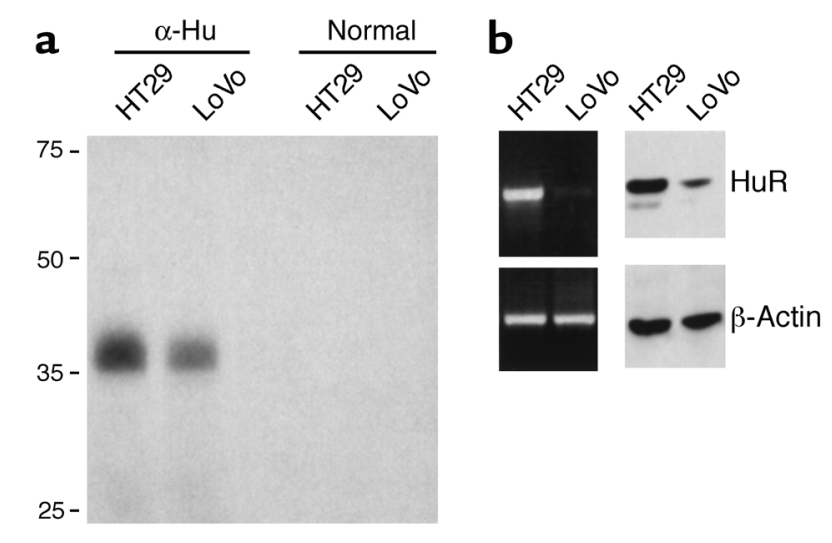

c

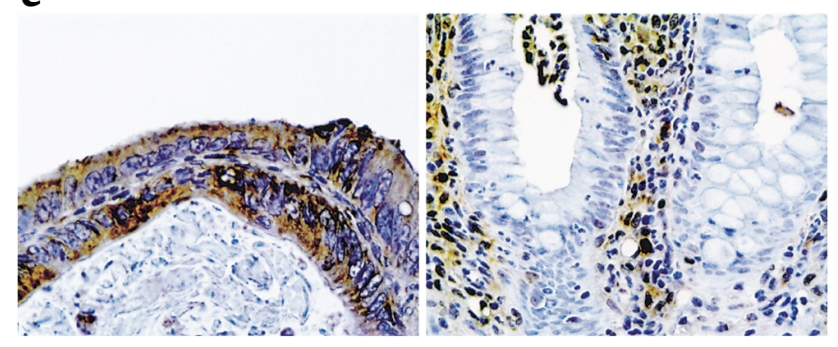

Figure 6

The HuR protein binds the COX-2 ARE. (a) UV-cross-linking reactions shown in Figure $5 c$ were immunoprecipitated using Ab's against HuR $(\alpha-H u)$. No cross-linked proteins were detected in control reactions using IgG from normal serum. (b) RT-PCR (left panel) and immunoblot (right panel) analysis of HuR expression from HT29 and LoVo cells. $\beta$-actin was detected as a control for RT-PCR and protein loading. (c) Immunohistochemical analysis of HuR expression in colorectal cancer (left) and normal tissue surrounding the tumor (right). No immunostaining of HuR was detected using Ab preabsorbed with recombinant HuR (data not shown). The results presented are representative of at least duplicate experiments.

cells was confirmed by analysis of proteins covalently cross-linked to the COX-2 ARE (Figure 5c). Polypeptides ranging from approximately 48 to 40 and $36 \mathrm{kDa}$ were predominately associated with the ARE in HT29 cells, whereas higher-molecular-weight proteins (90-60 $\mathrm{kDa}$ ) were bound in LoVo cells. These findings indicate that defects in posttranscriptional control reside in the altered capacity of trans-acting regulatory factors to interact with the COX-2 mRNA.

The HuR protein binds the COX-2 AU-rich element. HuR protein, an ubiquitously expressed member of the ELAV family of RNA-binding proteins, has been identified as a trans-acting factor involved in RNA-stabilization $(30,31)$. Based on the ability of HuR protein to bind AREs and to facilitate stabilization of rapidly degraded transcripts $(22,32,33)$, we hypothesized that this factor plays a role in regulating COX- 2 mRNA turnover. To test this idea, we determined if HuR interacts with the COX-2 ARE by immunoprecipitating HuR in UV-cross-linked cell lysates. As shown in Figure $6 \mathrm{a}$, immunoprecipitation assays using $\operatorname{Ig} G$ from high-titer anti-Hu autoantibodies $(24,34)$ identified a single radiolabeled polypeptide migrating at $36 \mathrm{kDa}$ in
HT29 and LoVo cell lysates. No cross-linked proteins were immunoprecipitated in identical reactions when nonimmune IgG was used. Differences in respective amounts of ARE-bound HuR were observed between HT29 and LoVo cells, with increased levels of crosslinked HuR protein (approximately fourfold) immunoprecipitated from HT29 cell lysates compared with those from LoVo cells based on an equal amount of protein loaded. The observed increased binding likely contributes to enhanced COX-2 mRNA stability seen in HT29 cells and is consistent with previous results showing similar stabilization of ARE-containing mRNAs by $\operatorname{HuR}(22,33)$.

Elevated expression of HuR mRNA and protein was detected in HT29 cells, whereas LoVo cells showed limited expression (Figure 6b). Similar results were seen in an RNase protection assay. As a control, we also tested for expression of the neural-restricted Hel$\mathrm{N} 1, \mathrm{HuD}$, and $\mathrm{HuC}$ mRNAs and did not detect them (data not shown). The expression of HuR protein in human colon tumors was examined by immunohistological analysis using representative tissue sections from adenocarcinoma and adjacent normal epithelium probed with an anti-HuR Ab (23) (Figure 6c). Similar to the localization of COX-2 seen in colon carcinomas $(7,35), \mathrm{HuR}$ expression was detected in neoplastic epithelial cells of the tumor (left panel) where the intensity of staining varied from strong to weak, indicating heterogeneous HuR expression within regions of the tumor. In contrast, adjacent normal colonic crypt epithelial cells displayed minimal immunoreactivity for HuR, although staining in a subpopulation of inflammatory cells within the lamina propria was observable (right panel). The expression of HuR within the carcinomatous epithelial cells may reflect its ability to influence tumor progression by promoting epithelial COX-2 expression during later stages of carcinogenesis (2).

Overexpression of HuR promotes enhanced expression of COX-2 and angiogenic factors in LoVo cells. Although the studies described above demonstrate that elevated levels of HuR are associated with cellular states displaying defective posttranscriptional regulation of COX-2, they do not address whether HuR is sufficient to increase COX-2 overexpression in colon cancer. Therefore, we sought to determine if overexpression of HuR could promote COX-2 and angiogenic factor expression in LoVo cells. To test this possibility, luciferase reporter constructs containing the COX-2 3'UTR were cotransfected into LoVo cells with a Flag-tagged HuR expression vector (pcDNA3-HuR-C-Flag) that was demonstrated previously to stabilize mRNAs containing class II AREs (22). As shown in Figure 7a, the COX-2 3'UTR inhibited luciferase expression in LoVo cells when cotransfected with the control vector pcDNA3. However, the ability of the COX-2 $3^{\prime}$ UTR to inhibit expression was silenced when HuR was overexpressed.

Since LoVo cells display constitutive transcription of COX-2, we next sought to determine if elevated HuR 
levels, as seen in HT29 cells, could promote endogenous gene expression. In HuR-transfected LoVo cells, enhanced COX-2 expression that correlated with HuR overexpression was detected by immunoblot analysis, whereas vector-transfected cells showed low levels of endogenous COX-2 (Figure 7b). These results were reflected in the ability of HuR-overexpressing LoVo cells to synthesize increased prostaglandin levels; an approximately twofold increase in $\mathrm{PGE}_{2}$ was produced in HuRtransfected LoVo cells (Figure 7c). The levels of the secreted angiogenic factors VEGF and IL-8 were similarly influenced in LoVo cells overexpressing HuR as compared with control transfections (Figure 7d). The ectopic overexpression of HuR in HT29 cells did not result in enhanced levels of COX-2, PGE 2 , VEGF, or IL-8 as compared with vector-transfected cells (data not shown).

\section{Discussion}

A variety of evidence gathered from epidemiological, whole animal, and in vitro cellular studies indicates that COX-2 expression is a rate-limiting step in colon cancer carcinogenesis. Although constitutive transcription of COX-2 may initiate unregulated expression of the enzyme in colon neoplasms (7), growing evidence implicates mRNA stability and translational efficiency as central controls in COX-2 expression (19, 20, 36-38). Previously, we showed that a major regulatory point of COX-2 gene expression occurs at the posttranscriptional level, and this control is mediated by the ARE-containing 3'UTR of the COX-2 mRNA (19, 20). The findings presented here substantiate the role of the COX-2 ARE in regulating COX-2 expression and demonstrate that cellular defects in the regulation of mRNA stability can contribute to elevated COX-2 protein expression, which accordingly promotes cell growth. These defects rest on the ability of RNA-binding proteins to properly interact with the COX-2 ARE and, based on these and other results $(22,33)$, that these interactions regulate the stability of COX- 2 and other angiogenic growth factor mRNAs.

Under normal cellular growth conditions, the expression of COX-2 is tightly controlled with rapid induction of COX-2 transcription occurring through a variety of stimuli (1). In colorectal neoplasia, a loss of transcriptional regulation has occurred leading to increased levels of COX-2 mRNA detected in virtually all colorectal tumors and cancer cell lines (6-8). Consistent with these findings we also found constitutive transcriptional activation of $C O X-2$ to be present in both LoVo and HT29 cells, with LoVo cells displaying increased COX-2 transcription as compared with HT29 cells (Figure 2b). This increased activation of the COX-2 promoter in LoVo cells, presumably a result of heightened ERK1/2 phosphorylation (Figure 4), did not accompany a concomitant increase in COX-2 protein. A lack of COX-2 protein could in part be attributed to efficient degradation of COX-2 mRNA and, given elevated COX-2 transcriptional activation, high levels of mRNA would be continually observed in LoVo cells. In contrast, the COX-2 mRNA is stabilized in HT29 cells and, as a consequence of altered posttranscriptional regulation, HT29 cells need only modest levels of COX-2 transcription. In addition, stabilized COX-2 mRNA was efficiently translated as detected by polysome fractionation analysis of samples from HT29 cells compared with LoVo cells (data not shown). These results provide insight into the observed variable expression of COX-2 protein in colon cancer tissues and cells $(11-14,39)$ and support recent findings that show increased COX-2 mRNA stability in colon cancer cells that constitutively express COX-2 (29).

Interestingly, HT29 cells had a similar ability to rapidly degrade c-myc mRNA as LoVo cells, suggesting a

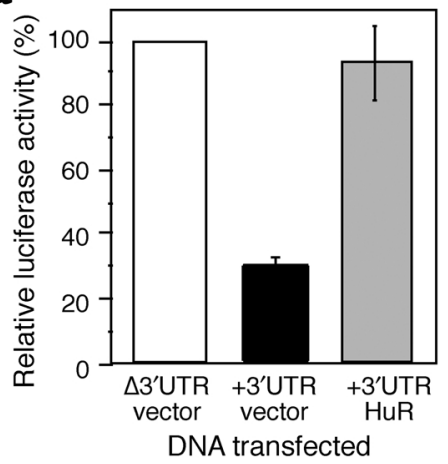

C

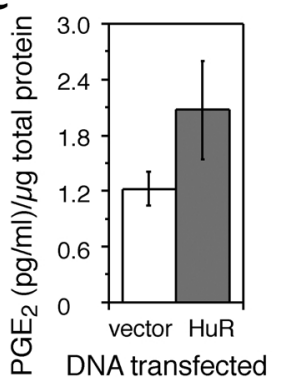

b

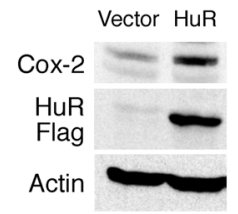

\section{Figure 7}

Overexpression of HuR in LoVo cells promotes enhanced expression of COX-2 and angiogenic factors. (a) Luciferase-reporter gene expression constructs containing the full-length COX-2 3'UTR $\left(+3^{\prime} U T R\right)$ or no $3^{\prime} U T R\left(\Delta 3^{\prime} U T R\right)$ were cotransfected with Flagtagged HuR (HuR) or control empty expression vector in LoVo cells. Relative activity was assessed as luciferase activity normalized to total protein. All percentages listed are based on expression of luciferase from the transfection of construct containing no 3'UTR with control vector ( $p c D N A 3)$ and is the average of three experiments done in triplicate. (b) Immunoblot of COX-2 protein in HuR or vector-transfected LoVo cell lysates. Flag-tagged HuR and $\beta$-actin were detected on the same blot as a control for transfection and protein loading. The data shown represent three experiments. (c) COX activity in HuR-transfected (filled bars) or vector-transfected (open bars) LoVo cells. PGE 2 levels were measured by ELISA in the media containing arachidonic acid and are the average of three experiments. (d) Expression of angiogenic factors VEGF (left panel) and IL-8 (right panel) in the culture media of HuR-transfected (filled bars) or vector-transfected (open bars) LoVo cells were measured by ELISA and are the average of three experiments. 
that defects in mRNA decay in HT29 cells are specific to the transcript. The rapidly degraded c-myc mRNA contains a class I ARE, which is characterized by dispersed AUUUA motifs in association with stretches of U-rich regions (40). In contrast, COX-2 mRNA contains a class II ARE, which has multiple copies of AUUUA motifs clustered together. The class II type is a feature of many growth factor mRNAs associated with angiogenesis. Thus, the inability to rapidly degrade class II-containing mRNAs also contributes to the overexpression of the angiogenic growth factors VEGF and IL-8, which share this type of motif. These and other ARE-containing growth factors have been shown to be upregulated in cancer cells that have altered posttranscriptional regulation of COX-2 $(18,29)$.

Control of mRNA decay is an effective way to regulate ARE-containing immediate-early genes. Findings demonstrating enhanced mRNA stability in tumor cells suggest that altered recognition of AU-rich sequences in neoplasia may lead to improper function of AREs $(41,42)$. Here we show that altered binding of cytoplasmic factors to the COX-2 ARE allows for enhanced expression of COX-2 and ARE-containing reporter gene constructs. We have identified HuR, a ubiquitously expressed member of the ELAV RNAbinding proteins, as a COX-2 ARE binding protein. As a result of elevated HuR protein expression owing to altered endogenous upregulation or ectopic overexpression, we conclude that increased expression of $\mathrm{HuR}$ lengthens mRNA half-lives and allows for enhanced mRNA translation $(31,43)$. These findings are consistent with previous results showing similar effects of HuR in vivo $(22,33)$ and define a role for aberrant $\mathrm{HuR}$ expression in colorectal neoplasia similar to other pathogenic states $(23,44,45)$.

The specific mechanisms by which AREs and their associated RNA-binding proteins function to promote rapid decay have been intensively studied (46). However, it is currently unclear how ARE-binding influences immediate-early gene mRNA degradation. Our data suggest that the limited ARE-binding of the highermolecular-weight complex (60-90 kDa) seen in HT29 cells (Figure 5c) reduces competition for COX-2 AREbinding sites. This, coupled with an increase in HuR expression, may result in enhanced binding of HuR to the COX-2 ARE. Through enhanced ARE-binding, HuR could indirectly inhibit mRNA deadenylation by promoting binding of the poly(A)-binding protein to the poly(A) tail (46). Alternatively, dysregulated HuR binding may impede the formation of a multiprotein complex involved with ARE-mediated destabilization. We are currently examining other factors that interact with the COX-2 ARE and have identified the RNAbinding proteins TIA- 1 and TIAR to be part of this complex (unpublished observations). Based on the ability of HuR to shuttle between the nucleus and cytoplasm (47), potential alterations in the HuR-accompanied transport of COX-2 mRNA from the nucleus to the cytoplasm in HT29 cells may also have direct impli- cations on COX-2 mRNA turnover and association with the translational apparatus (31).

The results presented here provide a novel link between the control of COX-2 expression at a posttranscriptional level and tumorigenesis. Based on these findings and others showing discrepancies between COX-2 mRNA levels and protein expression in the early stages of colon carcinogenesis (11), we suggest that loss of posttranscriptional regulation occurs during the transition from adenoma to carcinoma development. This, coupled with observations showing increases in COX-2 mRNA and protein levels, correlates with elevated tumor size and invasiveness $(4,48)$, implying that tumor progression is influenced by defects in regulation of COX-2 and angiogenic factor mRNA decay.

\section{Acknowledgments}

We thank Lualhati Harkins for technical assistance with the immunohistochemistry. The HuR mAb (mAb 3A2) and pcDNA3-HuR-C-Flag vector was kindly provided by I. Gallouzi and J. Steitz. We are grateful to P. Anderson, N. Kedersha, B. Preston, N. Deane, and H. Sheng for their helpful advice. E. Wilson and K. Ullman provided constructive comments on the manuscript. Grants from the American Heart Association (9930102N to D.A. Dixon), American Cancer Society (RPG-97-111-01-CCE to P.H. King), and National Cancer Institute (CA73992) supported this work. A National Cancer Institute Cancer Center Support Grant CA42014 supports the core facilities at the Huntsman Cancer Institute.

1. Williams, C.S., Mann, M., and DuBois, R.N. 1999. The role of cyclooxygenases in inflammation, cancer, and development. Oncogene. 18:7908-7916. 2. Prescott, S.M. 2000. Is cyclooxygenase- 2 the alpha and the omega in cancer? J. Clin. Invest. 105:1511-1513.

3. Prescott, S.M., and Fitzpatrick, F.A. 2000. Cyclooxygenase-2 and carcinogenesis. Biochim. Biophys. Acta. 1470:69-78.

4. Oshima, M., et al. 1996. Suppression of intestinal polyposis in $\mathrm{APC}^{\Delta 716}$ knockout mice by inhibition of cyclooxygenase 2 (COX-2). Cell. 87:803-809.

5. Reddy, B.S., et al. 2000. Chemoprevention of colon cancer by specific cyclooxygenase-2 inhibitor, celecoxib, administered during different stages of carcinogenesis. Cancer Res. 60:293-297.

6. Eberhart, C.E., et al. 1994. Up-regulation of cycloxygenase 2 gene expression in human colorectal adenomas and adenocarcinomas. Gastroenterology. 107:1183-1188.

7. Kutchera, W., et al. 1996. Prostaglandin synthase 2 is abnormally expressed in human colon cancer: evidence for a transcriptional effect. Proc. Natl. Acad. Sci. USA. 93:4816-4820.

8. Dimberg, J., Samuelsson, A., Hugander, A., and Soderkvist, P. 1998. Gene expression of cyclooxygenase-2, group II and cytosolic phospholipase A2 in human colorectal cancer. Anticancer Res. 18:3283-3287.

9. Williams, C.S., Tsujii, M., Reese, J., Dey, S.K., and DuBois, R.N. 2000. Host cyclooxygenase- 2 modulates carcinoma growth. J. Clin. Invest. 105:1589-1594.

10. Rigas, B., Goldman, I.S., and Levine, L. 1993. Altered eicosanoid levels in human colon cancer. J. Lab. Clin. Med. 122:518-523.

11. Kargman, S., et al. 1995. Expression of prostaglandin G/H synthase- 1 and -2 protein in human colon cancer. Cancer Res. 55:2556-2559.

12. DuBois, R.N., Radhika, A., Reddy, B.S., and Entingh, A.J. 1996. Increased cyclooxygenase-2 levels in carcinogen-induced rat colonic tumors. Gastroenterology. 110:1259-1262.

13. Sheng, H., et al. 1997. Inhibition of human colon cancer cell growth by selective inhibition of cyclooxygenase-2. J. Clin. Invest. 99:2254-2259.

14. Dimberg, J., Samuelsson, A., Hugander, A., and Soderkvist, P. 1999. Differential expression of cyclooxygenase 2 in human colorectal cancer. Gut. 45:730-732.

15. Prescott, S.M., and White, R.L. 1996. Self-promotion? Intimate connections between APC and prostaglandin H synthase-2. Cell. 87:783-786.

16. Tsujii, M., and DuBois, R.N. 1995. Alterations in cellular adhesion and 
apoptosis in epithelial cells overexpressing prostaglandin endoperoxide synthase 2. Cell. 83:493-501.

17. Tsujii, M., Kawano, S., and DuBois, R.N. 1997. Cyclooxygenase-2 expression in human colon cancer cells increases metastatic potential. Proc. Natl. Acad. Sci. USA. 94:3336-3340.

18. Tsujii, M., Kawano, S., Tsuji, S., Sawaoka, H., Hori, M., and DuBois, R.N 1998. Cyclooxygenase regulates angiogenesis induced by colon cancer cells. Cell. 93:705-716.

19. Dixon, D.A., Kaplan, C.D., McIntyre, T.M., Zimmerman, G.A., and Prescott, S.M. 2000. Post-transcriptional control of cyclooxygenase-2 gene expression. The role of the $3^{\prime}$-untranslated region. J. Biol. Chem. 275:11750-11757.

20. Sheng, H., et al. 2000. Transforming growth factor- $\beta 1$ enhances Ha-rasinduced expression of cyclooxygenase- 2 in intestinal epithelial cells via stabilization of mRNA. J. Biol. Chem. 275:6628-6635.

21. Gallouzi, I.E., et al. 2000. HuR binding to cytoplasmic mRNA is perturbed by heat shock. Proc. Natl. Acad. Sci. USA. 97:3073-3078.

22. Fan, X.C., and Steitz, J.A. 1998. Overexpression of HuR, a nuclear-cytoplasmic shuttling protein, increases the in vivo stability of ARE-containing mRNAs. EMBO J. 17:3448-3460.

23. Nabors, L.B., Gillespie, G.Y., Harkins, L., and King, P.H. 2001. HuR, an RNA stability factor, is expressed in malignant brain tumors and binds to adenine- and uridine-rich elements within the $3^{\prime}$ untranslated regions of cytokine and angiogenic factor mRNAs. Cancer Res. 61:2154-2161.

24. Nabors, L., Furneaux, H., and King, P. 1998. HuR, a novel target of anti$\mathrm{Hu}$ antibodies, is expressed in non-neural tissues. J. Neuroimmunol. 92:152-159.

25. Kawamori, T., Rao, C., Seibert, K., and Reddy, B. 1998. Chemopreventive activity of Celecoxib, a specific cyclooxygenase-2 inhibitor, against colon carcinogenesis. Cancer Res. 58:409-412.

26. Tegeder, I., Pfeilschifter, J., and Geisslinger, G. 2001. Cyclooxygenase-independent actions of cyclooxygenase inhibitors. FASEB J. 15:2057-2072

27. Marx, J. 2001. Anti-inflammatories inhibit cancer growth-but how? Science. 291:581-582.

28. Sheng, H., Shao, J., and DuBois, R.N. 2001. K-Ras-mediated increase in cyclooxygenase 2 mRNA stability involves activation of the protein kinase B. Cancer Res. 61:2670-2675.

29. Shao, J., Sheng, H., Inoue, H., Morrow, J.D., and DuBois, R.N. 2000. Regulation of constitutive cyclooxygenase- 2 expression in colon carcinoma cells. J. Biol. Chem. 43:33951-33956.

30. Brennan, C.M., and Steitz, J.A. 2001. HuR and mRNA stability. Cell. Mol. Life Sci. 58:266-277.

31. Keene, J. 1999. Why is Hu where? Shuttling of early-response messenger RNA subsets. Proc. Natl. Acad. Sci. USA. 96:5-7.

32. Ma, W.-J., Chung, S., and Furneaux, H. 1997. The ELAV-like proteins bind to AU-rich elements and to the poly(A) tail of mRNA. Nuc. Acids Res 25:3564-3569.

33. Peng, S.S.-Y., Chen, C.-Y.A., Xu, N., and Shyu, A.-B. 1998. RNA stabilization by the AU-rich element binding protein, HuR, an ELAV protein. EMBO J. 17:3461-3470.

34. King, P.H., Redden, D., Palmgren, J.S., Nabors, L.B., and Lennon, V.A. 1999. $\mathrm{Hu}$ antigen specificities of ANNA-I autoantibodies in paraneoplastic neurological syndromes. J. Autoimmun. 13:435-443.

35. Sano, H., et al. 1995. Expression of cyclooxygenase-1 and - 2 in human colorectal cancer. Cancer Res. 55:3785-3789.

36. Lasa, M., et al. 2000. Regulation of cyclooxygenase 2 mRNA stability by the mitogen-activated protein kinase p38 signaling cascade. Mol. Cell. Biol. 20:4265-4274.

37. Ristimaki, A., Garfinkel, S., Wessendorf, J., Maciag, T., and Hla, T. 1994. Induction of cyclooxygenase- 2 by interleukin-1 $\alpha$. J. Biol. Chem. 269:11769-11775.

38. Ristimaki, A., Narko, K., and Hla, T. 1996. Down-regulation of cytokineinduced cyclo-oxygenase- 2 transcript isoforms by dexamethasone: evidence for post-transcriptional regulation. Biochem. J. 318:325-331.

39. Sheng, H., Shao, J., Morrow, J.D., Beauchamp, R.D., and DuBois, R.N. 1998. Modulation of apoptosis and Bcl-2 expression by prostaglandin E2 in human colon cancer cells. Cancer Res. 58:362-366.

40. Xu, N., Chen, C.-Y.A., and Shyu, A.-B. 1997. Modulation of the fate of cytoplasmic mRNA by AU-rich elements: key sequence features controlling mRNA deadenylation and decay. Mol. Cell. Biol. 17:4611-4621.

41. Schuler, G.D., and Cole, M.D. 1988. GM-CSF and oncogene mRNA stabilities are independently regulated in trans in a mouse monocytic tumor. Cell. 55:1115-1122.

42. Ross, H.J., Santo, N., Ueyama, Y., and Koeffler, H.P. 1991. Cytokine messenger RNA stability is enhanced in tumor cells. Blood. 77:1787-1795.

43. Antic, D., and Keene, J.D. 1997. Embryonic lethal abnormal visual RNAbinding proteins involved in growth, differentiation, and posttranscriptional gene expression. Am. J. Hum. Genet. 61:273-278.

44. Levy, N.S., Chung, S., Furneaux, H., and Levy, A.P. 1998. Hypoxic stabilization of vascular endothelial growth factor mRNA by the RNA-binding protein HuR. J. Biol. Chem. 273:6417-6423.

45. Wang, W., Caldwell, M.C., Lin, S., Furneaux, H., and Gorospe, M. 2000. HuR regulates cyclin A and cyclin B1 mRNA stability during cell proliferation. EMBO J. 19:2340-2350.

46. Wilusz, C.J., Wormington, M., and Peltz, S.W. 2001. The cap-to-tail guide to mRNA turnover. Nat. Rev. Mol. Cell. Biol. 2:237-246.

47. Fan, X.C., and Steitz, J.A. 1998. HNS, a nuclear-cytoplasmic shuttling sequence in HuR. Proc. Natl. Acad. Sci. USA. 95:15293-15298.

48. Fujita, T., et al. 1998. Size- and invasion-dependent increase in cyclooxygenase 2 levels in human colorectal carcinomas. Cancer Res. 58:4823-4826. 